\title{
Circulating Hepatocellular Carcinoma Cells are Characterized by CXCR4 and MMP26
}

\author{
Chang Yu Zhiting Wang Xiaomei Xu Wei Xiang Xiaoying Huang \\ Department of Respiratory Medicine, the First Affiliated Hospital of Wenzhou Medical University, \\ Wenzhou, China
}

\section{Key Words}

Circulating tumor cells (CTCs) • Cancer stem cells (CSCs) • Hepatocellular carcinoma (HCC) • MMP26 • CXCR4

\begin{abstract}
Background/Aims: Primary hepatocellular carcinoma (HCC) is highly invasive, and often results in an early distal metastasis resulting in poor prognosis and therapeutic outcome. Cancer cells disseminating from the tumor and entering circulation are termed circulating tumor cells (CTCS). Although substantial progress has been made to identify those CTCs in HCC, no good marker (cocktail) has so far been identified. Methods: Since only tumorigenic CTCs form metastatic tumor in distal organs, we thus compared the HCC cells that form tumor spheres in culture to those that do not. We transduced HCC cells with a RFP reporter under MMP26 promoter and purified MMP26+CXCR4+ HCC cells. We examined tumor sphere formation in culture, presence of tumor cells in the circulation as well as capability of developing metastatic tumor after transplantation of MMP26+CXCR4+ HCC cells into nude mice, compared to other populations in HCC. Results: Sphere-forming HCC cells expressed high levels of MMP26 and CXCR4. MMP26+CXCR4+ HCC cells formed significantly more tumor spheres in culture, compared to MMP26-CXCR4-, MMP26-CXCR4+ or MMP26+CXCR4- HCC cells. Moreover, tumor cells were more frequently detected in the circulation when MMP26+CXCR4+ HCC cells were subcutaneously transplanted. Further, subcutaneous transplantation of MMP26+CXCR4+ HCC cells, but not transplantation of MMP26-CXCR4-, MMP26-CXCR4+ or MMP26+CXCR4HCC cells significantly developed distal metastatic tumors. Conclusion: MMP26+CXCR4+ cells may be CTCs in HCC. Selective elimination of MMP26+CXCR4+ cells may substantially reduce $\mathrm{HCC}$ metastasis after primary tumor resection.
\end{abstract}




\begin{tabular}{|c|c|c|}
\hline Cellular Physiology & Cell Physiol Biochem 2015;36:2393-2402 & \\
\hline and Biochemistrv & $\begin{array}{l}\text { DOI: 10.1159/000430201 } \\
\text { / Published online: July 27, } 2015\end{array}$ & $\begin{array}{l}\text { O } 2015 \text { S. Karger AG, Basel } \\
\text { www.karger.com/cpb }\end{array}$ \\
\hline
\end{tabular}

\section{Introduction}

Hepatocellular carcinoma (HCC) is a common and malignant tumor worldwide and is the third most lethal cancer, largely due to that HCC cells frequently invade vascular system at an early stage of the carcinogenesis and then metastasize through circulation to implant into distal organs [1-7]. Therefore, early detection and prevention of metastasis of HCC cells appear to be critical for developing effective therapy.

The past studies on the invasion and metastases of HCC cells have highlighted a pivotal role of circulating tumor cells (CTCs) in the process of cancer metastases [1-7]. CTCs are a particular population of cancer cells in the primary tumor. CTCs detach from the tumor mass and subsequently enter the blood circulation, from where they home to distal organs with a favorable environment for the tumor cells to stay and grow [1-7]. Since identification of CTCs in HCC may allow approaches to target these cells to inhibit cancer metastases, great efforts have been made to characterize CTCs in HCC [1-7]. Since only tumorigenic CTCs can form metastatic tumors in distal organs, CTCs often share some characteristics with cancer stem cells (CSCs), which are cancer cells that are responsible for cancer relapse and metastasis [111]. However, CSCs are not equivalent to CTCs, since not all CTCs form distal tumor, and not all CSCs undergo metastases [1-7].

So far, among all CTC surface markers, CXCR4, which is a unique receptor for stromal cell -derived factor-1 (SDF-1), has been shown to be the most important one, and has been used to characterize CTCs in different cancers, including HCC [12-20]. However, CXCR4 appears to be not sufficient to purify real CTCs, since CXCR4 is expressed in a certain number of HCC cells, and they are not all CTCs. Hence, there is an urgent need for better characterizing CTCs in HCC.

Matrix Metalloproteinases (MMPs) are a family of zinc endopeptidases that digest and break down extracellular matrix during embryonic development, tissue remodeling and carcinogenesis. MMP26, also known as endometase or matrilysin-2, is a member of MMPs, and is expressed predominantly in the placenta and uterus. Recently, MMP26 has been detected in a number of cancers [21-29]. However, the role of MMP26 in the carcinogenesis of HCC has not been studied.

Here, we compared the HCC cells that form tumor sphere in culture to those that do not. We transduced HCC cells with a RFP reporter under MMP26 promoter and purified MMP26+CXCR4+ HCC cells. We examined tumor sphere formation, presence of tumor cells in the circulation and capability of developing metastatic tumor after transplantation of MMP26+CXCR4+ HCC cells into nude mice, compared to other populations in HCC. We found that sphere-forming HCC cells expressed high levels of MMP26 and CXCR4. MMP26+CXCR4+ HCC cells formed significantly more tumor spheres in culture, compared to MMP26CXCR4-, MMP26-CXCR4+ or MMP26+CXCR4- HCC cells. Moreover, tumor cells were more frequently detected in the circulation when MMP26+CXCR4+ HCC cells were subcutaneously transplanted. Further, subcutaneous transplantation of MMP26+CXCR4+ HCC cells, but MMP26-CXCR4-, MMP26-CXCR4+ or MMP26+CXCR4- HCC cells significantly developed distal metastatic tumors.

\section{Materials and Methods}

Cell line culture and treatment

HepG2 is a human HCC line purchased from American Type Culture Collection (ATCC, Rockville, MD, USA), and was cultured in Dulbecco's modified Eagle's medium (DMEM, Invitrogen, Carlsbad, CA, USA) supplemented with 15\% fetal bovine serum (FBS; Sigma-Aldrich, St Louis, MO, USA) in a humidified chamber with $5 \% \mathrm{CO}_{2}$ at $37^{\circ} \mathrm{C}$.

Transduction of HCC cells

We used pcDNA3.1-CAG-GFP, pcDNA3.1-CAG-RFP and pcDNA3.1-CAG-luciferase plasmids as backbones (all from Clontech, Mountain View, CA, USA). The GFP coding sequence was digested with 
Xhol and BamHI and subcloned with a 2A into a pcDNA3.1-CAG-luciferase, resulting in a construct named pCAG-luciferase-2A-GFP. The small 2A peptide sequences, when cloned between genes, allow for efficient, stoichiometric production of discrete protein products within a single vector through a novel "cleavage" event within the 2A peptide sequence. Human MMP26 promoter was cloned by PCR, using human genomic DNA as a template. MMP26 promoter was cloned into pcDNA3.1-CAG-RFP to replace CAG promoter, resulting in a construct named pMMP26-RFP. Finally, pCAG-luciferase-2A-GFP and pMMP26-RFP were connected into one construct. Sequencing was performed to confirm the correct orientation of the new plasmid. To generate lentiviral particles, HEK293T cells were seeded in a $100 \mathrm{~mm}$ dish at $5 \times 10^{4} \mathrm{cells} / \mathrm{cm}^{2}$ and cotransfected with $10 \mu \mathrm{g}$ of prepared plasmid and $5 \mu \mathrm{g}$ each of packaging plasmids (REV, pMDL and VSV-G) using Lipofectamine-2000 (Invitrogen). The supernatant containing lentiviral particles was collected 48 hours after transfection and filtered through a $0.45 \mu \mathrm{m}$ syringe filter. The viruses were purified using $\mathrm{CsCl}$ density centrifugation and then titered by a quantitative densitometric dot-blot assay. For cell transduction in vitro, $\mathrm{HCC}$ cells were seeded in $100 \mathrm{~mm}$ plates at $1.5 \times 10^{4} \mathrm{cells} / \mathrm{cm}^{2}$ one day prior to lentiviral infection. The lentiviral particles were added along with $10 \mu \mathrm{g} / \mathrm{ml}$ polybrene (Sigma-Aldrich) to the cell culture at a multiplicity of infection (MOI) of 100 for 48 hours. Then the cells were washed twice with complete media and purified for transduced bells based on GFP by flow cytometry. Some MMP26+ cells are yellow due to co-expression of RFP and GFP.

\section{Primary Tumor Sphere Culture}

Purified tumor cells by flow cytometry were washed, acutely dissociated in oxygenated artificial cerebrospinal fluid and subject to enzymatic dissociation. Tumor cells were then resuspended in tumor sphere media (TSM) consisting of a serum-free DMEM, human recombinant EGF (20ng/ml; Sigma-Aldrich), bFGF (20ng/ml; Sigma-Aldrich), leukemia inhibitory factor (10ng/ml; Sigma-Aldrich) and N-acetylcysteine $\left(60 \mu \mathrm{g} / \mathrm{ml}\right.$; Sigma-Aldrich), and then plated at a density of $2 \mathrm{X} 10^{6}$ cells $/ 60 \mathrm{~mm}$ plate.

\section{Analysis of CXCR4, RFP and GFP by flow cytometry}

CXCR4, RFP and GFP-based cell analysis and sorting were performed by flow cytometry, after the cultured cells or cells from circulation were labeled with APC-conjugated CXCR4 antibodies (BectonDickinson Biosciences, San Jose, CA, USA). GFP and RFP were determined by direct fluorescence. Flow cytometry was performed using a FACSAria (Becton-Dickinson Biosciences) flow cytometer. Negative controls were applied to remove background noise and to confirm positive cells. Data were analyzed and quantified using Flowjo software (Flowjo LLC, Ashland, OR, USA).

\section{Cell growth assay}

For assay of cell growth, cells were seeded into 24 well-plate at $1 \mathrm{X} 10^{4}$ cells per well and subjected to a Cell Proliferation Kit (MTT, Roche, Indianapolis, IN, USA), according to the instruction of the manufacturer. The MTT assay is a colorimetric assay for assessing viable cell number, taking advantage that NADPH-dependent cellular oxidoreductase enzymes in viable cells reduce the tetrazolium dye 3-(4,5-dimethylthiazol-2-yl)2,5-diphenyltetrazolium bromide (MTT) to its insoluble formazan in purple readily being quantified by absorbance value (OD) at $570 \mathrm{~nm}$. Experiments were performed 5 times.

\section{Quantitative real-time PCR (RT-qPCR)}

Total RNA were extracted from cells with RNeasy kit (Qiagen, Hilden, Germany), for cDNA synthesis. Quantitative real-time PCR (RT-qPCR) was performed in duplicates with QuantiTect SYBR Green PCR Kit (Qiagen). All primers were purchased from Qiagen. Data were collected and analyzed with the Rotorgene software accompanying the PCR machine, using 2- $\Delta \Delta \mathrm{Ct}$ method for quantification of the relative mRNA expression levels. Values of genes were first normalized against $\alpha$-tubulin, and then compared to controls.

\section{Western blot}

The protein was extracted from the HCC cells by RIPA buffer (Sigma, St Louis, USA) for Western Blot. The supernatants were collected after centrifugation at $1.2 \times 10^{4} \mathrm{~g}$ at $4^{\circ} \mathrm{C}$ for $20 \mathrm{~min}$. Protein concentration was determined using a BCA protein assay kit (Bio-rad, China), and whole lysates were mixed with $4 \times$ SDS loading buffer $(125 \mathrm{mmol} / \mathrm{L}$ Tris-HCl, 4\% SDS, $20 \%$ glycerol, $100 \mathrm{mmol} / \mathrm{L}$ DTT, and $0.2 \%$ bromophenol blue) at a ratio of 1:3. Samples were heated at $100^{\circ} \mathrm{C}$ for $5 \mathrm{~min}$ and were separated on SDS-polyacrylamide 
gels. The separated proteins were then transferred to a PVDF membrane. The membrane blots were first probed with a primary antibody. After incubation with horseradish peroxidase-conjugated second antibody, autoradiograms were prepared using the enhanced chemiluminescent system to visualize the protein antigen. The signals were recorded using X-ray film. Primary antibodies were anti-CXCR4, anti-MMP26 and anti- $\alpha$-tubulin (all purchased from Santa Cruz Biotechnology, Dallas, Texas, USA).

Mouse handling

All mouse experiments were approved by the Institutional Animal Care and Use Committee at the First Affiliated Hospital of Wenzhou Medical University (Animal Welfare Assurance). Mice were kept in a Specific pathogen fee (SPF) condition. Surgeries were performed in accordance with the Principles of Laboratory Care, supervised by a qualified veterinarian. All efforts were made to minimize pain and suffering. Female 10 week-old nude mice were used in the current study. Ten mice were analyzed in each experimental condition.

In vivo transplantations of tumor cells and bioluminescence imaging

Purified cells $\left(10^{6}\right)$ were subcutaneously (s.c.) injected into the nude mice. The tumor growth after 4 months was monitored and quantified by luminescence levels. Bioluminescence was measured with the IVIS imaging system (Xenogen Corp., Alameda, CA, USA). All of the images were taken 10 minutes after intraperitoneal injection of luciferin (Sigma-Aldrich) of $150 \mathrm{mg} / \mathrm{kg}$ body weight, as a 60 -second acquisition and 10 of binning. During image acquisition, mice were sedated continuously via inhalation of 3\% isoflurane. Image analysis and bioluminescent quantification was performed using Living Image software (Xenogen Corp).

Statistical analysis

All statistical analyses were carried out using the SPSS 18.0 statistical software package. All values in cell and animal studies are depicted as mean \pm standard deviation and are considered significant if $\mathrm{p}<0.05$. All data were statistically analyzed using one-way ANOVA with a Bonferoni correction, followed by Fisher's Exact Test to compare two groups.

\section{Results}

HCC cells that form tumor sphere express high MMP26 and CXCR4

We used a human HCC cell line, HepG2, in our study. Since only tumorigenic CTCs form metastatic tumor in distal organs, we compared the HCC cells that form tumor sphere in culture (sphere+) to those that do not (sphere-) (Fig. 1A). We found that sphere-forming HCC cells expressed significantly higher levels of MMP26 and CXCR4, by RT-qPCR (Fig. 1B), and by Western blot (Fig. 1C). These data prompted us to examine whether MMP26+CXCR4+ cells may be a specific subpopulation of CXCR4+ cells that are responsible for HCC distal metastases.

Labeling of HCC cells

To allow tracing tumor formation in living mice and isolation of MMP26+ tumor cells from mice, we transduced the HepG2 cells with a lentivirus carrying luciferase and GFP reporter under the control of a CAG promoter, and RFP reporter under MMP26 promoter (Fig. 2A). The transduced cells were purified based on GFP expression by flow cytometry. In culture, the transduced HCC cells were all green, and some cells appeared to be yellow (both red and green) due to co-expression of MMP26 (Fig. 2B). These transduced HepG2 cells were re-analyzed by flow cytometry, showing that the yellow MMP26+ cells represent about 3.5\% of total HCC cells (Fig. 2C).

MMP26+CXCR4+ HCC cells grow much faster than negative populations

Since MMP26 has been recently shown to play a critical role in the carcinogenesis of some cancers, and since CXCR4 is a well-known CTC marker, we hypothesized MMP26+CXCR4+ HCC cells may be more purified CTCs than CXCR4+ cells in HCC. Thus, we separated HCC cell 
Fig. 1. HCC cells that form tumor sphere express high MMP26 and CXCR4. (A) We studied the HCC cells from a human HepG2 HCC cell line and compared the cells that form tumor sphere in culture (sphere+) to those that do not (sphere-). (B-C) The sphere-forming HCC cells expressed significantly higher levels of MMP26 and

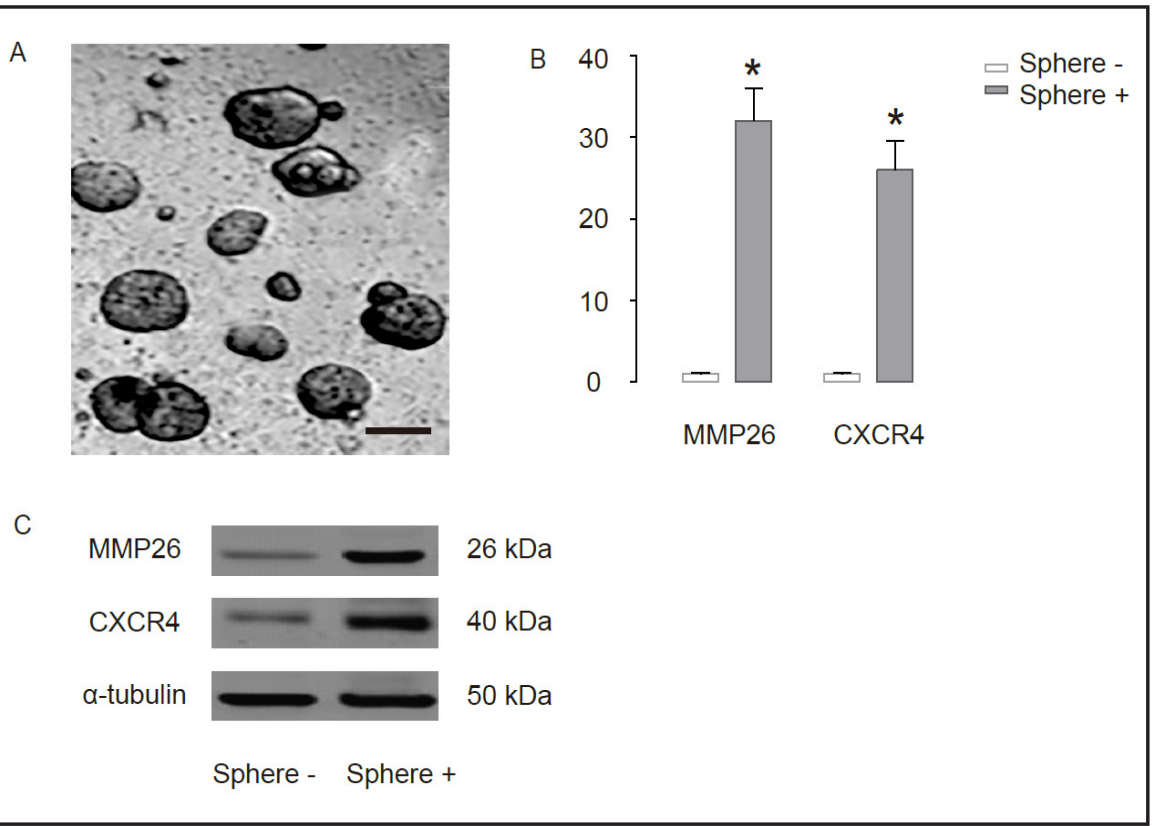
CXCR4, by RT-qPCR (B), and by Western blot (C). N=5. *p<0.05. Statistics: one-way ANOVA with a Bonferoni correction, followed by Fisher's Exact Test to compare two groups. Scale bar is $50 \mu \mathrm{m}$.

Fig. 2. Labeling of HCC cells. (A) To allow tracing tumor formation in living mice and isolation of tumor cells from MMP26+ tumor cells from mice, we transduced the HepG2 cells with a lentivirus carrying luciferase and GFP reporter under the control of a CAG promoter, and RFP reporter under MMP26 promoter. (B) The purified transduced cells are all GFP+, and some cells appeared to be yellow

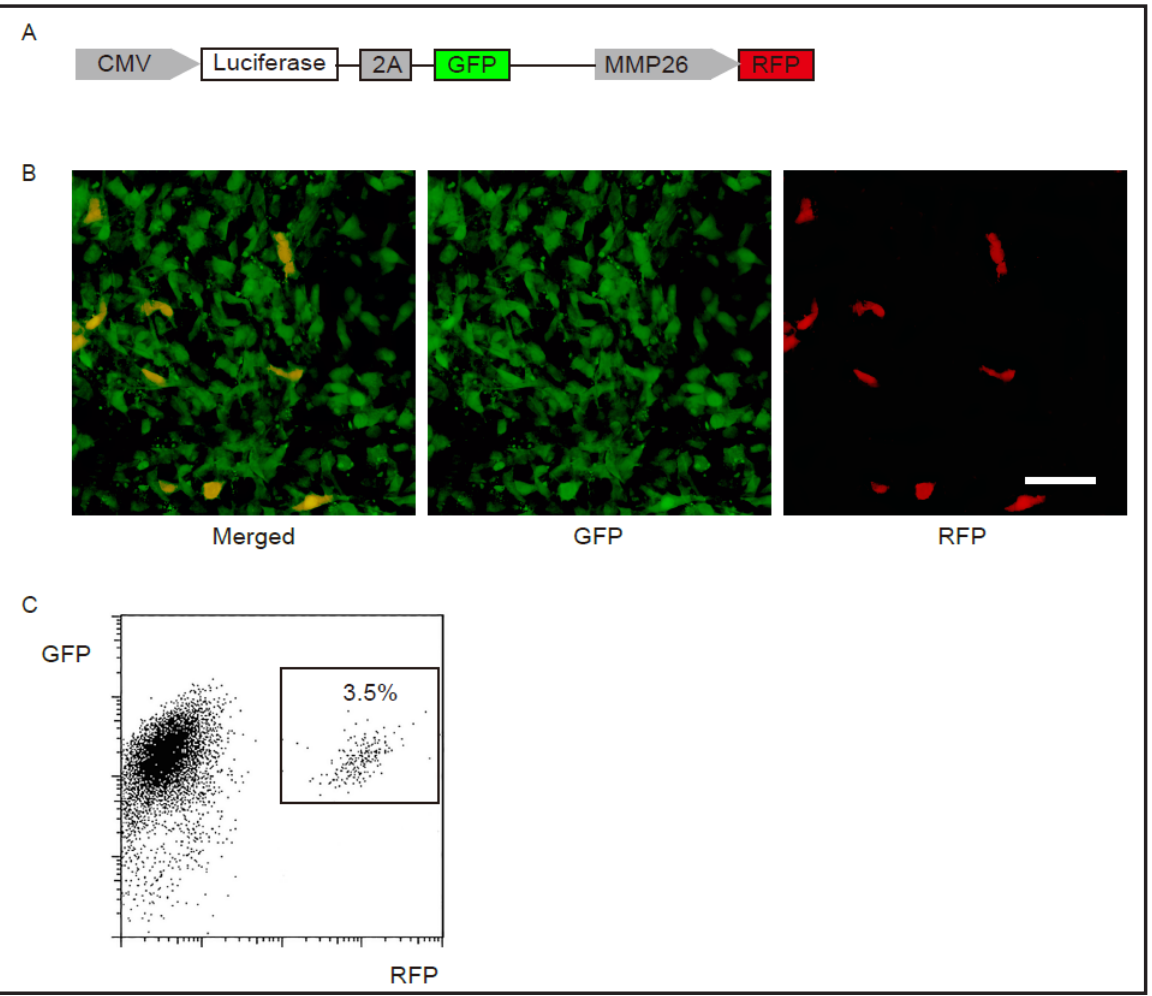
(both red and green) due to co-expression of MMP26. (C) The transduced HepG2 cells were re-analyzed by flow cytometry, showing by a representative flow chart. Rectangle: GFP+RFP+ cells. Scale bar is $20 \mu \mathrm{m}$.

subpopulations based on MMP26 and CXCR4 expression by flow cytometry (Fig. 3A). Among all HCC cells, about $12.4 \%$ cells were MMP26-CXCR4+, about 3.5\% cells were MMP26+CXCR4-, 


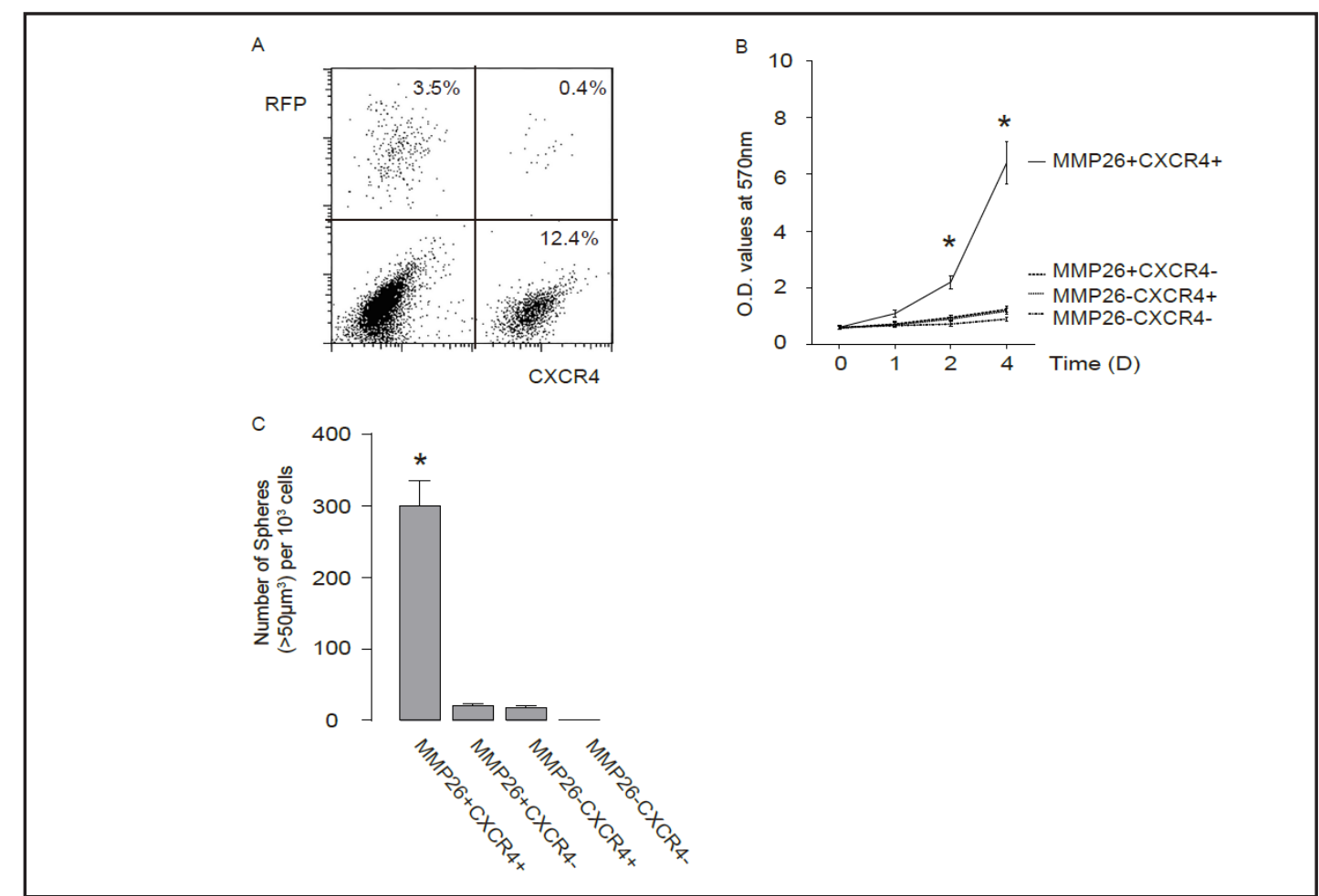

Fig. 3. MMP26+CXCR4+ HCC cells grow much faster than negative populations. (A) HCC cell subpopulations were separated based on MMP26 (RFP) and CXCR4 expression by flow cytometry. (B) MTT assay for all 4 subpopulations of HCC cells. (C) The 4 subpopulations of HCC cells were treated with the tumor sphere media (TSM), and the formation of tumor sphere was quantified. $N=5$. ${ }^{*} \mathrm{p}<0.05$. Statistics: one-way ANOVA with a Bonferoni correction, followed by Fisher's Exact Test to compare two groups.

and about $0.4 \%$ cells were MMP26+CXCR4+ (Fig. 3A). All 4 subpopulations of HCC cells were applied in a MTT assay to evaluate cell growth. We found that MMP26+CXCR4+ cells grew significantly faster than the other 3 subpopulations (Fig. 3B). Moreover, when we challenged these 4 subpopulations of HCC cells with the tumor sphere media (TSM), we found that MMP26+CXCR4+ cells generated significantly more sphere-like structures than either MMP26-CXCR4+ or MMP26+CXCR4- cells, while MMP26-CXCR4- cells did not form spherelike structures at all (Fig. 3C). Together, these data suggest that MMP26+CXCR4+ HCC cells are tumor-forming CSC-like cells.

MMP26+CXCR4+ HCC cells are CTCs

Then we examined the presence of GFP+ tumor cells in the circulation and capability of developing metastatic tumor after transplantation of these 4 subpopulations into nude mice. First, we checked the presence of GFP+ cells in circulation by flow cytometry, shown by representative flow charts of a negative sample from a mouse that received MMP26-CXCR4+ HCC cells (Fig. 4A), and a positive sample from a mouse that received MMP26+CXCR4+ HCC cells (Fig. 4B). While we hardly detected GFP+ circulatory cells in the mice that received either MMP26-CXCR4+, MMP26+CXCR4-, or MMP26-CXCR4- HCC cells, we did detect GFP+ circulatory cells in most of the mice that received MMP26+CXCR4+ HCC cells (Fig. 4C). Moreover, we also evaluated the incidence of the development of tumors in distal organs [lung (Fig. 5A, blue arrow, mouse in the middle) and liver (Fig. 5A, red arrow, mouse in the right)], 4 months after transplantation. We found significant higher frequency for the formation of distal tumors in mice transplanted with MMP26+CXCR4+ HCC cells, compared to the other 3 subpopulations (Fig. 5B). Together, these data suggest that MMP26+CXCR4+ cells are CTC cells in HCC. 
Fig. 4. Detection of circulatory tumor cells after transplantation. We examined the presence of GFP+ tumor cells in the circulation and capability of developing metastatic tumor after transplantation of these 4 subpopulations into nude mice. (A-B) Representative flow charts of a negative sample from a mouse that received MMP26-CXCR4+ HCC cells (A), and a positive sample from a mouse that received MMP26+CXCR4+ HCC cells (B). (C) A summary of detection of GFP+ circulatory tumor cells. $\mathrm{N}=10 . \quad{ }^{*} \mathrm{p}<0.05$. Statistics: one-way ANOVA with a Bonferoni correction, followed by Fisher's Exact Test to compare two groups.
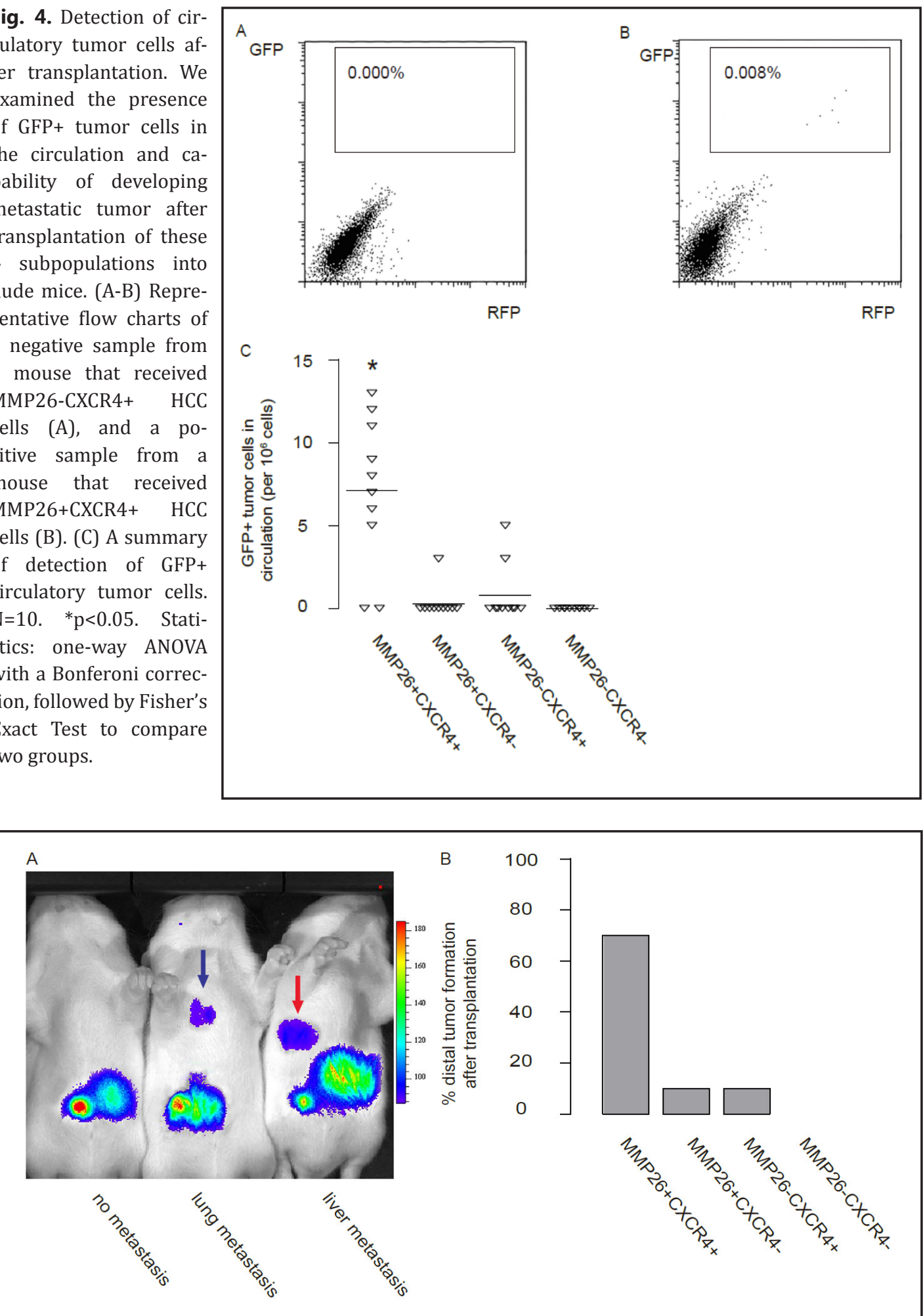

Fig. 5. Incidence of the development of tumors in distal organs. We evaluated the incidence of the development of tumors in distal organs by bioluminescence imaging. (A) A Representative image showing no metastasis (mouse in the left), lung metastasis (blue arrow, mouse in the middle) and liver metastasis (red arrow, mouse in the right), 4 months after transplantation. (B) A summary of frequency for the formation of distal tumors in mice transplanted with 4 subpopulations of HCC cells. 


\section{Discussion}

Understanding the molecular regulation of HCC metastases is extremely important for improving the therapeutic outcome. Recently, the acknowledgement of the role of CTCs in cancer metastases sheds light on the development of innovative HCC-associated treatments. Efficient manipulation of CTCs requires precise characterization of this population among all cancer cells. To date, isolation of CTCs from HCC is mainly dependent on cell surface markers, among which the most important one is CXCR4. SDF-1/CXCR4 axis is a potential chemoattractant system for regulation of cell migration and homing, and plays an important and unique role in the regulation of stem/progenitor/cancer cell trafficking [30-34]. CXCR4 is known to be expressed on some tumor cells, which may metastasize to the organs that secrete/express SDF-1 [30-34]. SDF-1 exerts pleiotropic effects regulating metastasisassociated processes, including cancer cell locomotion, chemoattraction and adhesion, and tumor vascularization [30-34]. Although CXCR4 has been shown to substantially enrich the CTC population in various cancers, it is noteworthy that the purification of CTCs by the mere use of CXCR4 is not optimized and the enrichment of CTCs by CXCR4 is limited [12-18]. Thus, identification of additional CTC markers for HCC is highly needed.

Here, we used a human HCC cell line, HepG2, in our study. We have also performed similar analyses on other HCC cell lines and essentially got similar results. Thus, our findings should not be cell-line dependent. In order to trace tumor cells in living animals as well as identification of tumor cells from the circulation in receipt mice, we labeled the cells with both luciferase and GFP reporter under the control of a CAG promoter. In addition, the MMP26+ cells were labeled with RFP and thus appeared to be yellow due to co-expression of GFP and RFP. Since GFP construct is behind a 2A following luciferase, all sorted GFP+ cells should express luciferase. Thus, no luciferase-negative cells were transplanted.

We separated HCC cells into 4 subpopulations based on MMP26 and CXCR4 expression and in HCC cells, and only $0.4 \%$ cells were MMP26+CXCR4+, which was much lower, compared to MMP26-CXCR4+ cells. The tumorigenic property of MMP26+CXCR4+ cells were examined by cell growth assay and caner sphere-like structure formation. Next, we examined CTC properties of these 4 subpopulations, and only detected tumor cells in the circulation of mice that received MMP26+CXCR4+ HCC cells with high frequency. Interestingly, all detected GFP+ tumor cells were all red, suggesting that CTCs express MMP26, which is consistent with findings in our in vitro study. In addition, high-frequent tumor formation in distal organs was exclusively detected in mice that received MMP26+CXCR4+ HCC cells. Taken together, these experiments suggest that MMP26+CXCR4+ cells are CTC cells in HCC.

To our knowledge, our study should be the first one to use a combination of MMP26 and CXCR4 to characterize CTCs in HCC. Since a role of MMP26 has not been studied in HCC, our study suggests that MMP26 may play a potential role in HCC-related pathogenesis. Future studies may address the molecular pathways that activate MMP26 as well as be regulated by MMP26 in HCC. MMP26+CXCR4+ cells may be a promising therapeutic target for HCC.

\section{Disclosure Statement}

The authors have declared that no competing interests exist.

\section{Reference}

1 Sainz B Jr, Heeschen C: Standing out from the crowd: Cancer stem cells in hepatocellular carcinoma. Cancer Cell 2013;23:431-433.

2 Chiba T, Kamiya A, Yokosuka O, Iwama A: Cancer stem cells in hepatocellular carcinoma: Recent progress and perspective. Cancer Lett 2009;286:145-153. 
3 Chiba T, Kanai F, Iwama A, Yokosuka O: Circulating cancer stem cells: A novel prognostic predictor of hepatocellular carcinoma. Hepatobiliary Surg Nutr 2013;2:4-6.

4 Nagano H, Ishii H, Marubashi S, Haraguchi N, Eguchi H, Doki Y, Mori M: Novel therapeutic target for cancer stem cells in hepatocellular carcinoma. J Hepatobiliary Pancreat Sci 2012;19:600-605.

5 Tian H, Huang P, Zhao Z, Tang W, Xia J: Hif-1alpha plays a role in the chemotactic migration of hepatocarcinoma cells through the modulation of cxcl6 expression. Cell Physiol Biochem 2014;34:15361546.

6 Zhang YS, Chu JH, Cui SX, Song ZY, Qu XJ: Des-gamma-carboxy prothrombin (dcp) as a potential autologous growth factor for the development of hepatocellular carcinoma. Cell Physiol Biochem 2014;34:903-915.

7 Jiang HL, Xu D, Yu H, Ma X, Lin GF, Ma DY, Jin JZ: Dax-1 inhibits hepatocellular carcinoma proliferation by inhibiting beta-catenin transcriptional activity. Cell Physiol Biochem 2014;34:734-742.

8 Petersson M, Niemann C: Stem cell dynamics and heterogeneity: Implications for epidermal regeneration and skin cancer. Curr Med Chem 2012;19:5984-5992.

9 Perez-Losada J, Balmain A: Stem-cell hierarchy in skin cancer. Nat Rev Cancer 2003;3:434-443.

10 Singh SR: Stem cell niche in tissue homeostasis, aging and cancer. Curr Med Chem 2012;19:5965-5974.

11 Singh SK, Clarke ID, Terasaki M, Bonn VE, Hawkins C, Squire J, Dirks PB: Identification of a cancer stem cell in human brain tumors. Cancer Res 2003;63:5821-5828.

12 Yang ZF, Ngai P, Ho DW, Yu WC, Ng MN, Lau CK, Li ML, Tam KH, Lam CT, Poon RT, Fan ST: Identification of local and circulating cancer stem cells in human liver cancer. Hepatology 2008;47:919-928.

13 Chen X, Lingala S, Khoobyari S, Nolta J, Zern MA, Wu J: Epithelial mesenchymal transition and hedgehog signaling activation are associated with chemoresistance and invasion of hepatoma subpopulations. J Hepatol 2011;55:838-845.

14 Lingala S, Cui YY, Chen X, Ruebner BH, Qian XF, Zern MA, Wu J: Immunohistochemical staining of cancer stem cell markers in hepatocellular carcinoma. Exp Mol Pathol 2010;89:27-35.

15 Piao LS, Hur W, Kim TK, Hong SW, Kim SW, Choi JE, Sung PS, Song MJ, Lee BC, Hwang D, Yoon SK: Cd133+ liver cancer stem cells modulate radioresistance in human hepatocellular carcinoma. Cancer Lett 2012;315:129-137.

16 Zhang J, Luo N, Luo Y, Peng Z, Zhang T, Li S: Microrna-150 inhibits human cd133-positive liver cancer stem cells through negative regulation of the transcription factor c-myb. Int J Oncol 2012;40:747-756.

17 Haraguchi N, Ishii H, Mimori K, Tanaka F, Ohkuma M, Kim HM, Akita H, Takiuchi D, Hatano H, Nagano H, Barnard GF, Doki Y, Mori M: Cd13 is a therapeutic target in human liver cancer stem cells. J Clin Invest 2010;120:3326-3339.

18 Yang ZF, Ho DW, Ng MN, Lau CK, Yu WC, Ngai P, Chu PW, Lam CT, Poon RT, Fan ST: Significance of cd90+ cancer stem cells in human liver cancer. Cancer Cell 2008;13:153-166.

19 Winkelman C: Inactivity and inflammation: Selected cytokines as biologic mediators in muscle dysfunction during critical illness. AACN clinical issues 2004;15:74-82.

20 Gillies K, Wertman J, Charette N, Dupre DJ: Anterograde trafficking of cxcr4 and ccr2 receptors in a prostate cancer cell line. Cell Physiol Biochem 2013;32:74-85.

21 Xu X, Ma J, Li C, Zhao W, Xu Y: Regulation of chondrosarcoma invasion by mmp26. Tumour Biol 2015;36:365-369.

22 Suomela S, Koljonen V, Skoog T, Kukko H, Bohling T, Saarialho-Kere U: Expression of mmp-10, mmp-21, mmp-26, and mmp-28 in merkel cell carcinoma. Virchows Arch 2009;455:495-503.

23 Li D: Peaking of mmp-26 and timp-4 marks invasive transition in prostate cancer. Cell Res 2006;16:741.

24 Lee S, Desai KK, Iczkowski KA, Newcomer RG, Wu KJ, Zhao YG, Tan WW, Roycik MD, Sang QX: Coordinated peak expression of mmp-26 and timp-4 in preinvasive human prostate tumor. Cell Res 2006;16:750-758.

25 Yamamoto H, Vinitketkumnuen A, Adachi Y, Taniguchi H, Hirata T, Miyamoto N, Nosho K, Imsumran A, Fujita M, Hosokawa M, Hinoda Y, Imai K: Association of matrilysin-2 (mmp-26) expression with tumor progression and activation of mmp-9 in esophageal squamous cell carcinoma. Carcinogenesis 2004;25:2353-2360.

26 Marchenko ND, Marchenko GN, Weinreb RN, Lindsey JD, Kyshtoobayeva A, Crawford HC, Strongin AY: Betacatenin regulates the gene of mmp-26, a novel metalloproteinase expressed both in carcinomas and normal epithelial cells. Int J Biochem Cell Biol 2004;36:942-956. 


\section{Cellular Physiology Cell Physiol Biochem 2015;36:2393-2402 \begin{tabular}{ll|l} 
and Biochemistry $\begin{array}{l}\text { DOI: 10.1159/000430201 } \\
\text { Published online: July 27, 2015 }\end{array}$ & $\begin{array}{l}\text { O 2015 S. Karger AG, Basel } \\
\text { www.karger.com/cpb }\end{array}$ \\
\cline { 2 - 3 }
\end{tabular} \\ Yu et al.: Identification of CTCs in HCC}

27 Zhou J, Lu S, Yang S, Chen H, Shi H, Miao M, Jiao B: Microrna-127 post-transcriptionally downregulates sept7 and suppresses cell growth in hepatocellular carcinoma cells. Cell Physiol Biochem 2014;33:15371546.

28 Hu X, Zhang F, Liu XR, Wu YT, Ni YM: Efficacy and potential microrna mechanism for computed tomography-guided percutaneous radiofrequency ablation of primary lung cancer and lung metastasis from liver cancer. Cell Physiol Biochem 2014;33:1261-1271.

29 Li H, Li Y, Liu D, Sun H, Liu J: Mir-224 is critical for celastrol-induced inhibition of migration and invasion of hepatocellular carcinoma cells. Cell Physiol Biochem 2013;32:448-458.

30 Powell AA, Talasaz AH, Zhang H, Coram MA, Reddy A, Deng G, Telli ML, Advani RH, Carlson RW, Mollick JA, Sheth S, Kurian AW, Ford JM, Stockdale FE, Quake SR, Pease RF, Mindrinos MN, Bhanot G, Dairkee SH, Davis RW, Jeffrey SS: Single cell profiling of circulating tumor cells: Transcriptional heterogeneity and diversity from breast cancer cell lines. PLoS One 2012;7:e33788.

31 Fusi A, Liu Z, Kummerlen V, Nonnemacher A, Jeske J, Keilholz U: Expression of chemokine receptors on circulating tumor cells in patients with solid tumors. J Transl Med 2012;10:52.

32 Li CX, Shao Y, Ng KT, Liu XB, Ling CC, Ma YY, Geng W, Fan ST, Lo CM, Man K: Fty720 suppresses liver tumor metastasis by reducing the population of circulating endothelial progenitor cells. PLoS One 2012; 7:e32380.

33 Charles J, Di Domizio J, Salameire D, Bendriss-Vermare N, Aspord C, Muhammad R, Lefebvre C, Plumas J, Leccia MT, Chaperot L: Characterization of circulating dendritic cells in melanoma: Role of ccr6 in plasmacytoid dendritic cell recruitment to the tumor. J Invest Dermatol 2010;130:1646-1656.

34 Lee K, Qian DZ, Rey S, Wei H, Liu JO, Semenza GL: Anthracycline chemotherapy inhibits hif-1 transcriptional activity and tumor-induced mobilization of circulating angiogenic cells. Proc Natl Acad Sci U S A 2009;106:2353-2358. 